\title{
Super lattice formation of an array of volatile wetting droplets
}

\author{
R. Burghaus \\ Institut für Theoretische Physik IV \\ Heinrich-Heine-Universität Düsseldorf \\ Universitätsstr. 1 \\ D-40225 Düsseldorf, Germany
}

\begin{abstract}
For an ordered array of critical volatile wetting droplets the formation of a super lattice by an Ostwald-ripening like competition process is considered. The underlying diffusion problem is treated within a quasistatic approximation and to first order in the inverse droplets distance. The approach is rather general but a square lattice and a triangular lattice are studied explicitly. Dispersion relations for the super-lattice growth of these arrays are calculated.
\end{abstract}

In a recent experiment C. Schäfle et al [1] nucleated diethylene glycol droplets out of a supersaturated vapor phase on a hexagonal array of hydrophilic (alkanethiol) patches on a hydrophobic substrate. The pressure of the system was reduced afterwards so that the droplets started to shrink by evaporation. During this process the hexagonal lattice split into two triangular super lattices in a sense that the droplets on one super lattice shrank faster than those on the other super lattice. The super lattice of faster shrinking droplets disappeared, finally. The remaining triangular droplet lattice appeared to be stable against further development of super structures. The appearance of the triangular super lattice from the hexagonal lattice is interpreted as an Ostwald-ripening type process. The system reduces its free energy by having few large droplets instead of many small [2, 3, 何. Similar observations where made by the same authors [1] as well as by Lacasta et al [5] simulating CahnHilliard dynamics of droplet arrays in a concentration field. The study of super lattice growth on an array of wetting droplets is not only of fundamental interest as an example of pattern formation and the dynamics of wetting on structured surfaces, but it is also important to learn about the features of volatile droplet arrays since they can be used to build e.g. chemical sensors (see [6, 7]).

The scope of this paper is the analytic discussion of the diffusional growth properties of a set of wetting droplets sitting on hydrophilic patches on a hydrophobic substrate. We are considering circular patches of equal radius $a$. Square and triangular lattice configurations of the patches will be studied explicitly. It is assumed that there is a wetting droplet sitting in the center of each patch. The different possible equilibrium configurations and phase transitions in such a system were discussed in great detail by Lenz and Lipowsky only recently [8, 9].

To discuss the diffusional growth of a wetting droplet out of the surrounding gas phase, one has to specify the boundary conditions of the diffusion equation on the substrate and on the droplets surface. Since there is no diffusion flux into the substrate, the normal derivative of the concentration field vanishes there. The concentration $c_{s}$ in the gas phase on top of the droplet is given by the Gibbs-Thomson relation. It is a constant along the droplets surface and is just a function of the radius of curvature $R$ 田 10

$$
c_{s}(R)=c_{0}\left(1+\frac{\Lambda}{R}\right),
$$

where $c_{0}$ is the concentration above a flat condensate and $\Lambda$ is a capillary length. 
We assume that the droplet is growing slowly due to its high density compared to the surrounding gas phase. The diffusion equation, which describes the concentration around the droplet, can be approximated by the Laplace-equation $\Delta c=0$, therefore. The Neumann condition of vanishing normal derivative on the substrate suggests to mirror the system on the substrate to fulfill the condition implicitly. This way the semi-infinite system becomes an infinite system formally and the spherical-cap shaped wetting droplet becomes a symmetric lens that provides a uniform (Dirichlet) boundary condition to the outside concentration field. Since this field is described by a Laplace equation, one can use an electrostatic analogy where the electrical potential corresponds to the concentration field. The mirrored droplet, i.e. the symmetric lens, is a conductor in this picture [4. 10, 11]. The volume growth $\dot{\Omega}$ of an individual droplet corresponds to the charge of the analogous conductor because the diffusion flux density on the droplets surface is given by the normal derivative of the concentration field and the charge density of the conductor is given by the electric field on top of the conductor which is the derivative of the potential. This argument leads to the growth equation

$$
\dot{\Omega}=C(R, \theta) \cdot\left(c_{\infty}-c_{s}(R)\right)
$$

eventually [4, 10], where $C(R, \theta)$ is the electric capacity of the symmetric lens and $c_{\infty}$ is the systems concentration far away from the droplet. The capacity $C(R, \theta)$ depends linearly on the radius of curvature $R$ and is a function of the wetting droplets contact angle $\theta$. It can be found in the literature $4,11,12$.

Since the surface tension between the droplet and the hydrophilic patch is smaller than the one between the droplet and the hydrophobic substrate, the contact (Youngs) angles are different in these regions. A small droplet, which completely sits on a hydrophilic patch, has a small contact angle $\theta_{1}$. If the droplet is too big to fit onto the patch it reaches out on the hydrophobic region where its contact angle $\theta_{2}$ is bigger. Consequently one can distinguish three different volume regimes for the droplets. Regime (1), where the contact line is on the hydrophilic patch completely, regime (2), where the contact line is fixed on the border between the hydrophilic and the hydrophobic material and regime (3), where the contact line is completely in the hydrophobic region (see fig. 11).

In regime (2) droplet growth takes place with a fixed contact line but a changing contact angle $\theta$. If $\theta<\pi / 2$, this leads to the unusual case that the radius of curvature $R$ decreases with an increasing droplet volume. This implies, that in this case a droplet in an environment $c_{\infty}=c_{s}(R)$ is not critical but stable because its Gibbs-Thomson boundary concentration increases with increasing volume unlike the usual case where the Gibbs-Thomson boundary condition decreases with increasing droplet size. This stability allows to grow droplets of identical size on an array of equally sized patches in the regime (2), which is important for experimental purposes.

Now there is not only one droplet in the system but the collective behavior of a system of droplets shall be studied. The boundary condition on top of every single droplet is given by (1), so that we can use the full electrostatic analogy again. We have a system of conductors where the relation between the charges and the potentials is given by a capacitance matrix which now yields

$$
\dot{\Omega}_{i}=\sum_{j} C_{i j} \cdot\left(c_{\infty}-c_{s}\left(R_{j}\right)\right)
$$

for the growth rates of the individual droplets generalizing eq. (2). Here the indexes $i, j$ count the individual droplets, $\Omega_{i}$ and $R_{i}$ are the volume and the radius of curvature of droplet $i$ and the matrix $C_{i j}$ is the abovementioned capacitance matrix of the system.

In the following we will assume that the lattice constant $d$ of the droplet array is large compared to the droplet sizes $\left(R_{i} \ll d\right)$. Then, the inverse of the capacitance matrix can be written

$$
C_{i j}^{-1}= \begin{cases}C_{i}^{-1}(R, \theta)+\mathcal{O}\left(1 / d^{2}\right) & \text { for } i=j \\ 1 / R_{i j}+\mathcal{O}\left(1 / d^{2}\right) & \text { for } i \neq j\end{cases}
$$

where $C_{i}(R, \theta)$ is the capacity of the droplet $i$ alone. $R_{i j}$ is the distance between the centers of the droplets $i$ and $j$. Eqs. (11), (3) and (4) lead to

$$
c_{0}\left(1+\frac{\Lambda}{R_{i}}\right)=c_{\infty}+\frac{1}{C_{i}\left(R_{i}, \theta_{i}\right)} \dot{\Omega}_{i}+\sum_{j \neq i} \frac{1}{R_{i j}} \dot{\Omega}_{j}
$$


to linear order in inverse droplet distances.

If we now consider a system where each droplet satisfies $R_{i}=R_{c}:=\left(\Lambda c_{0}\right) /\left(c_{\infty}-c_{0}\right)$ (i.e. it is critical if its radius increases with increasing volume or stable if its radius decreases with increasing volume), $\dot{R}_{i}=0$ is a (in most cases unstable) solution of the growth equation. To study the onset of the spontaneous development of a super lattice on the regular lattice of wetting droplets, we introduce $\delta R_{i} \cdot e^{\omega t}:=R_{i}-R_{c}$ where the $\delta R_{i}$ describe an eigenmode of the linearized version of eq. (5). The growth exponent reads

$$
\omega=\frac{-c_{0} \frac{\Lambda}{R_{c}^{3}}}{2 \Omega^{\prime}\left[C_{c}+\sum_{j \neq i} \frac{1}{R_{i j}} \frac{\delta R_{j}}{\delta R_{i}}\right]} .
$$

Here $C_{c}$ is the capacity of an individual critical droplet alone and $\Omega^{\prime}:=d \Omega(R, \theta(R)) /\left.d R\right|_{R=R_{c}}$ describes the variation of the droplet volume when its radius $R$ is changed obeying the physical boundary conditions on the contact angle, i.e. holding the contact angle constant if we are in the regime 1 or 3 of fig. 11 and pinning the contact line (varying the contact angle) if we are in the regime 2 . As mentioned earlier, $\Omega^{\prime}$ is negative in regime 2 if $\theta<\pi / 2$.

The sum $\Sigma:=d \cdot \sum_{j \neq i}\left(1 / R_{i j}\right)\left(\delta R_{j} / \delta R_{i}\right)$ is independent of the choice of $i$ since we look at eigenmodes of eq. (5). It depends on the type of droplet lattice and the mode under consideration. One finds a divergence at $\Sigma / d=-C_{c}$. This divergence is an artifact of the approximation for the capacitance matrix to first order in $1 / R_{i j}$, i.e. neglecting $\mathcal{O}\left(1 / d^{2}\right)$. The fastest growing mode is found at the smallest $\Sigma$. In the following the growth exponent $\omega$ will be evaluated for the different modes of an infinite square lattice and an infinite triangular lattice.

Due to the symmetry of the square lattice the eigenmodes of the growth equation are just Fourier modes. In fig. 2 the sum $\Sigma$ is plotted as a function of $m$ and $n$, where $m, n$ are the wavenumbers along the two axis of the square lattice. The minimum is found at $\Sigma(\pi, \pi)$ which means that the preferred growth mode leads to a checkerboard structure. The two corresponding square super lattices on the original square lattice carry uniformly shrinking or uniformly growing droplets, respectively. This behavior is optimal in the sense that every growing droplet is surrounded only by shrinking droplets and the other way around leading to a fast exchange of matter.

In case of a triangular lattice things are not as obvious since the geometry does not allow every droplet to be surrounded by droplets of a different growth sign without frustration. If we introduce two non rectangular axis along two of the primary axis of the triangular lattice we have again translational symmetry along these axis and can Fourier analyze the system. We get a sum $\Sigma$ for the corresponding wavenumbers as shown in fig. 3. The dispersion relation has a threefold degenerate minimum at $(\pi, \pi),(0, \pi)$ and $(\pi, 0)$ reflecting the special symmetry of the triangular lattice as shown in fig. 4 . These fastest eigenmodes can be characterized by the property that each droplet has four nearest neighbors with opposite growth signs and two nearest neighbors with the same growth sign. In this respect the growth conditions in the triangular lattice are inferior to those in the square lattice. This is reflected by the fact that the minimum $\Sigma$ of the square lattice is lower than the one of the triangular lattice, i.e. the super lattice formation is faster in the square lattice case..

C. Schäfle et al. found in their experiment [1] that the triangular droplet lattice that occurred as a super lattice of the initial hexagonal lattice did not show any further instability, whereas the calculations provided in this paper display an instability as sketched in fig. 14 Unlike the subject of this paper the experiment does not analyze critical droplets so that in addition to $\omega$ there is a timescale related to the overall evaporation of droplets. It seems reasonable to expect that the instability of the triangular droplet lattice could be seen experimentally by stretching the timescale provided by the overall evaporation, i.e. by going closer to the critical concentration.

I like to thank C. Schäfle et al. for sharing their experimental results with me prior to publication. I'm especially grateful to B. Schmittmann and R. K. P. Zia for their kind hospitality and helpful discussions. This work is supported by the Deutsche Forschungsgemeinschaft via BU 1172/1-1 and "Benetzung und Strukturbildung an Grenzflächen" as well as the EU via FMRX-CT 98-0171 "Foam Stability and Wetting Transitions". 


\section{References}

[1] C. Schäfle et al. (to appear in Phys. Rev. Lett.)

[2] I. M. Lifshitz, V. V. Slyozov, J. Phys. Chem. Solids 19, 35 (1961)

[3] C. Wagner, Z. Elektrochem. 65, 581 (1961)

[4] R. Burghaus, Phys. Rev. E 54, 6955 (1996)

[5] A. M. Lacasta, I. M. Sokolov, J. M. Sancho and F. Sagués, Phys. Rev. E, 57, 6198 (1998)

[6] A. Kumar et al., Acc. Chem. Res. 28, 219 (1995)

[7] F. Morhard et al., Proc. ECS 97-19, 1058 (1997)

[8] P. Lenz and R. Lipowsky, Phys. Rev. Lett. 80, 1920 (1998)

[9] P. Lenz, "Benetzungsphänomene auf strukturierten Substraten", PhD-thesis (1998)

[10] R. Burghaus cond-mat/9903107

[11] R. G. Picknett, R. Bexon, J. Coll. Interf. Sci. 61 , 336 (1977)

[12] C. Snow, J. of Research NBS, 43, 377 (1949)

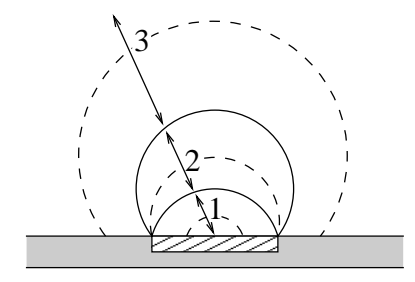

Figure 1: Three qualitatively different droplet sizes can be distinguished: (1) A droplet which sits on the hydrophilic patch completely; (2) the contact line of the droplet sits on the edge of the hydrophilic region; (3) the contact line is on the hydrophobic substrate completely.

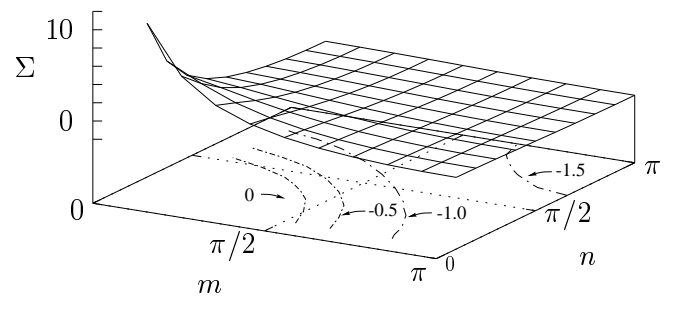

Figure 2: The sum $\Sigma$ as a function of the wavenumbers $m$ and $n$ for a square lattice.

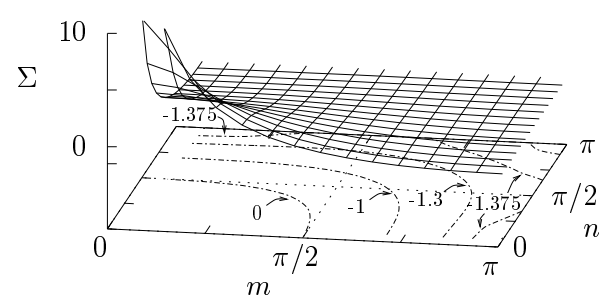

Figure 3: The sum $\Sigma$ as a function of the wavenumbers $m$ and $n$ for a triangular lattice.

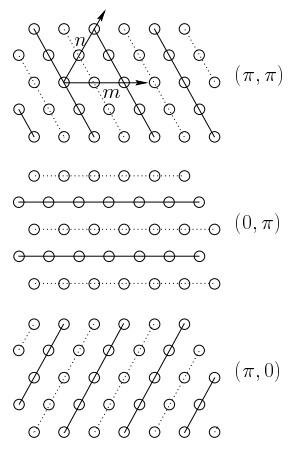

Figure 4: The fastest growing modes of a triangular lattice. 